\title{
MULTIPLICITY RESULTS FOR CRITICAL FRACTIONAL EQUATIONS WITH SIGN-CHANGING WEIGHT FUNCTIONS
}

\author{
YANG PU* AND JIA-FENG LIAO
}

Abstract. In this paper, we consider a time-independent fractional equation:

$$
\begin{cases}(-\Delta)^{s} u=f(x)|u|^{2_{s}^{*}-2} u+g(x)|u|^{q-1} u, & x \in \Omega ; \\ u=0, & x \in R^{N} \backslash \Omega,\end{cases}
$$

where $\Omega$ is a smooth bounded domain, $s \in(0,1), N>2 s, 0<q<1$, the coefficient functions $f$ and $g$ may change sign. We first obtain the existence of ground state solution by the Nehari method under the combined effect of coefficient functions. Then we find the multiplicity of positive solutions by Mountain pass theorem under some stronger conditions, and one of them is a ground state solution.

Mathematics subject classification (2010): 35A15, 35R11, 35J60, 47G20.

Keywords and phrases: Fractional laplacian equations, variational techniques, critical growth.

\section{REFERENCES}

[1] N. LANDKOF, Foundations of mordern potrntial theory, Springer-Verlag, New York, 1972.

[2] H. BeRESTYCKI, P.-L. LiONS, Nonlinear scalar field equations, I. Existence of a ground state, Arch. Rational Mech. Anal, 82 (1983), 313-345.

[3] N. LAS KIn, Fractional quantum mechanics and Lévy path integrals, Phys. Lett. 268 (2000), 298-305.

[4] D. Applebaum, Lévy Processes and Stochastic Calculus, seconded, Camb. Stud. Adv. Math., vol. 116, Cambridge University Press, Cambridge, 2009.

[5] J. Bertoin, Lévy Processes, Camb. Tracts Math., vol. 121, Cambridge University Press, Cambridge, 1996.

[6] R. Cont, P. Tankov, Financial Modelling with Jump Processes, Chapman \& Hall/CRC Financ. Math. Ser., Chapman and Hall/CRC, Boca Raton, FL, 2004.

[7] L. Vlahos, H. Isliker, Y. Kominis, K. Hizonidis, Normal and anomalous diffusion: a tutorial, in: T. Bountis (Ed.), Order and Chaos, vol. 10, Patras University Press, 2008.

[8] J. G. TAN, The Brezis-Nirenberg type problem involving the square root of the Laplacian, Calc. Var. Partial Differ. Equ. 42 (2011), 21-41.

[9] B. Barrios, E. Colorado, A. De Pablo, U. SÁnchez, On some critical problems for the fractional Laplacian operator, J. Differential Equations, 252 (2012), 6133-6162.

[10] A. Fiscella, G. Molica Bisci, R. Servadei, Bifurcation and multiplicity results for critical nonlocal fractional Laplacian problems, Bull. Sci. Math. 140 (2016), 14-35.

[11] N. Kouhestani, H. Mahyar, A. Moameni, Multiplicity results for a non-local problem with concave and convex nonlinearities, Nonlinear Anal. 182 (2019), 263-279.

[12] P. L. LI, H. R. SUN, Existence results and bifurcation for nonlocal fractional problems with critical Sobolev exponent, Comp. Math. Appl. 78 (2019), 1720-1731.

[13] O. H. Miyagaki, D. Motreanu, F. R. Pereira, Multiple solutions for a fractional elliptic problem with critical growth, J. Differential Equations, 269 (2020), 5542-5572.

[14] A. Ambrosetti, H. Brézis, G. Cerami, Combined effects of concave and convex nonlinearities in some elliptic problems, J. Funct. Anal. 122 (1994), 519-543. 
[15] R. ServadeI, E. VAldinoci, The Brezis-Nirenberg result for the fractional Laplacian, Trans. Amer. Math. Soc. 367 (2015), 67-102.

[16] R. SERVADEI, The Yamabe equation in a non-local setting, Adv. Nonlinear Anal. 2 (2013), 235-270.

[17] R. SERVADEI, A critical fractional Laplace equation in the resonant case, Topol. Methods Nonlinear Anal. 43 (2014), 251-267.

[18] R. SERVADEI, E. VAldinoci, A Brezis-Nirenberg result for non-local critical equations in low dimension, Commun. Pure Appl. Anal. 12 (2013), 2445-2464.

[19] B. Barrios, E. Colorado, R. SERVAdei, F. Soria, A critical fractional equation with concaveconvex power nonlinearities, Ann. I. H. Poincaré. 32 (2015), 875-900.

[20] W. J. Chen, S. B. Deng, The Nehari manifold for nonlocal elliptic operators involving concaveconvex nonlinearities, Z. Angew. Math. Phys. 66 (2015), 1387-1400.

[21] A. QUAAS, A. L. XIA, Multiple positive solutions for nonlinear critical fractional elliptic equations involving sign-changing weight functions, Z. Angew. Math. Phys. 67 (2016), Art. 40, 21 pp.

[22] Q. F. WANG, The Nehari manifold for a fractional Laplacian equation involving critical nonlinearities, Commun. Pure Appl. Anal. 6 (2018), 2261-2281.

[23] C. M. Chu, J. J. Sun, H. M. Suo, Multiplicity of positive solutions for critical fractional equation involving concave-convex nonlinearities and sign-changing weight functions, Mediterr. J. Math. 13 (2016), 4437-4446.

[24] W. ChEn, C. L. TANG, Infinitely many for critical fractional equation with sign-changing weight function, J. Appl. Anal. Comput. 10 (2020), 131-139.

[25] G. Tarantello, On nonhomogeneous elliptic equations involving critical Sobolev exponent, Ann. I. H. Poincaré. 3 (1992), 281-304.

[26] E. Colorado, A. De Pablo, U. SÁnchez, Perturbations of a critical fractional equation, Pacific J. Math. 271 (2014), 65-85.

[27] A. Cotsiolis, N. TAvoularis, Best constants for Sobolev inequalities for higher order fractional derivatives, J. Math. Anal. Appl. 295 (2004), 225-236.

[28] X. CABRÉ, J. DÁvila, Nonlinear equations for fractional Laplacians I: Regularity, maximum principles and Hamiltonian estimates, Ann. I. H. Poincaré. 31 (2014), 23-53.

[29] X. Ros-Oton, J. Serra, The Dirichlet problem for the fractional Laplacian: Regularity up to the boundary, J. Math. Pures Appl. 101 (2014), 275-302.

[30] H. CYCON, R. Frose, W. KIRSCH, Schrödinger Operators with Application to Quantumn Mechanics and Global Geometry, Berlin: Spriger, 1987.

[31] L. Evans, Partial Differential Equations, Amer. Math. Soc., Providence, Rhode Island, 1998.

[32] M. WILlEM, Minimax Theorems, Birthäuser, Boston, 1996.

[33] H. BRÉZIS, E. LIEB, A relation between pointwise convergence of functions and convergence of functionals, Proc. Amer. Math. Soc. 88 (1983), 486-490.

[34] I. Ekeland, On the variational principle, J. Math. Anal. Appl. 47 (1974), 324-353.

[35] H. Royden, P. FitzPatrick, Real Analysis, Prentice Hall, Upper Saddle River, 2007. 